\title{
Tratamento conservador de prolapso de órgão pélvico com pessário: revisão de literatura
}

\section{Pessary use for conservative treatment of pelvic organ prolapse: literature review}

\author{
Cristiane Regina de Barros ${ }^{1}$, Rogério Bonassi Machado², \\ Ana Carolina Marchesini de Camargo ${ }^{3}$, Thomaz Rafael Gollop ${ }^{4}$
}

Barros CR, Bonassi Machado RB, Camargo ACM, Gollop TR. Tratamento conservador de prolapso de órgão pélvico com pessário: revisão de literatura / Pessary use for conservative treatment of pelvic organ prolapse: literature review. Rev Med (São Paulo). 2018 mar.-abr.;97(2):154-9.

RESUMO: INTRODUÇÃO: O prolapso de órgãos pélvicos (POP) sintomático afeta a qualidade de vida (QV) das mulheres e demanda tratamento. Por ser mais prevalente em pacientes idosas seu tratamento cirúrgico pode ser limitado por contraindicações clínicas ou desejo da paciente por um tratamento conservador; e por isso estas pacientes poderão se beneficiar com o uso de pessários. OBJETIVO: analisar os trabalhos publicados referentes ao impacto na qualidade de vida $(\mathrm{QV})$ das mulheres com POP através do tratamento com pessário, bem como os fatores descritos como risco para o insucesso deste método. MÉTODO: revisão bibliográfica utilizando as Bases de Dados Scientific Electronic Library On-line (SciELO) e PubMed", com os termos "pelvic organ prolapse, pessary and quality of life" publicados entre janeiro de 2011 a dezembro de 2016. RESULTADO: Os estudos mostram que o tratamento com pessário apresenta boa evolução, melhorando a sintomatologia causada pelo prolapso, semelhantes às pacientes que foram submetidas à tratamento cirúrgico. Os questionários para avaliação de QV utilizados foram diversificados, mas todos observaram melhora. CONCLUSÃO: o tratamento conservador com pessário é uma opção viável para mulheres com POP mesmo em estadio avançado, mas é mais utilizado naquelas nas quais há contraindicação para tratamento cirúrgico.

Descritores: Pessários; Prolapso de órgão pélvico; Qualidade de vida; Literatura de revisão como assunto.

\begin{abstract}
INTRODUCTION: Symptomatic pelvic organ prolapse (POP) affects the quality of life (QoL) of women and requires treatment. Because it is more prevalent in elderly patients, its surgical treatment may be limited by clinical contraindications or the patient's desire for conservative treatment. These patients may, therefore, benefit from the use of pessaries. PURPOSE: to analyse the published studies concerning the impact on the quality of life $(\mathrm{QoL})$ of women with POP through the use of pessary treatment, as well as the risk factors arising from the failure of this method. METHOD: bibliographic review using the Scientific Electronic Library Online (SciELO) and PubMed ${ }^{\circledR}$ databases, using the terms "pelvic organ prolapse, pessary and quality of life", published between January 2011 and December 2016. RESULT: The studies show that treatment using a pessary reports good progress, improving the symptomatology caused by the prolapse, and this result is similar to patients who underwent surgical treatment. The questionnaires for the evaluation of QoL used were diverse, but all observed improvement. CONCLUSION: conservative pessary treatment is a viable option for women suffering from POP, even at an advanced stage of POP, but a pessary is more commonly used by those in whom there is contraindication to surgical treatment.
\end{abstract}

Keywords: Pessaries; Pelvic organ prolapsed; Quality of life; Review literature as topic.

1. Fisioterapeuta especialista em obstetrícia e mestranda no Programa de Pós-Graduação em Ciências da Saúde da Faculdade de Medicina de Jundiaí, Centro Universitário Nossa Senhora do Patrocínio - Itu, São Paulo, SP. Email: crisbarros_fisio@hotmail.com. ORCID: 0000-0002-1382-8080.

2. Professor Adjunto do Departamento de Obstetrícia e Ginecologia da Faculdade de Medicina de Jundiaí, Faculdade de Medicina de Jundiaí, Jundiaí, SP. Email: rogeriobonassi@terra.com.br. ORCID: 0000-0001-9361-0905.

3. Professor Adjunto do Departamento de Obstetrícia e Ginecologia da Faculdade de Medicina de Jundiaí, Jundiaí, SP. Email: anacamarquesini@gmailcom. ORCID: 0000-0003-2716-9693.

4. Professor Associado do Departamento de Obstetrícia e Ginecologia da Faculdade de Medicina de Jundiaí, Coordenador do Serviço de Assoalho Pélvico do Hospital Universitário de Jundiá, Jundiaí, SP. Email: trgollop@usp.br. ORCID: 0000-0001-9841-9501.

Endereço para correspondência: C. R. Barros. Rua Durval Savioli, 35. Condomínio Aldea D’Espana - Chácara Bela Vista, Itu, SP, Brasil. 


\section{INTRODUÇÃO}

$\mathrm{O}$ prolapso de órgãos pélvicos (POP) é definido pela Sociedade Internacional de Continência (ICS) como uma procedência de parede vaginal anterior, posterior ou da cúpula vaginal. Acomete mulheres, principalmente idosas e apresenta como principais fatores de risco a multiparidade, parto, doenças do colágeno, fatores hereditários, obesidade, fumo, menopausa e obstipação ${ }^{1}$. Apesar de não ser uma doença que leve ao óbito, ela tem um grande impacto na QV dessas pacientes ${ }^{2}$.

Segundo o Instituto Brasileiro de Geografia e Estatística (IBGE) estima-se que em 2050 teremos mais de 9 milhões de mulheres com 80 anos ou mais ${ }^{3}$ e por isso com o aumento da expectativa de vida mais casos de POP surgirão nos próximos anos. O tratamento desta patologia é fundamental para restabelecer as atividades diárias e consequentemente melhora da $\mathrm{QV}^{2}$.

Os casos de POP assintomáticos não demandam qualquer tratamento, mas quando geram alterações na função urinária ou intestinal, nas atividades cotidianas de locomoção, laborais ou na atividade sexual, ou simplesmente impedem que a paciente possa sentar-se confortavelmente o tratamento é imperativo.

Os prolapsos de órgãos pélvicos são classificados em 4 graus: I, II, III e IV. O ponto de referência para a classificação dos prolapsos é o anel himenal. Os prolapsos de órgãos pélvicos mais graves (III e IV) se exteriorizam por vários centímetros além do anel himenal.

A maioria das mulheres com POP são submetidas a correções cirúrgicas por ser um tratamento definitivo, enquanto o pessário é apenas paliativo.

Apesar da cirurgia trazer resultados positivos, o procedimento torna-se contraindicado em casos de instabilidades clínicas e comorbidades ${ }^{2}$. Nas situações onde a paciente não tenha condições clínicas para a cirurgia ou tenha preferência por um tratamento não invasivo, pode-se optar pelo pessário ${ }^{4}$. Este dispositivo de silicone comumente é utilizado na forma de anel ou donut, devendo ser individualmente adaptado de acordo com a avaliação do quociente de prolapso de órgãos pélvicos (POP-Q) e do diâmetro da vagina para escolha do tamanho do mesmo e habitualmente é indicado para pacientes com prolapsos estadios grau 3 ou 4 .

Os pessários vaginais são escolhidos em sua forma por exemplo: anel ou Donut (similar ao doce) e em seu tamanho de acordo com a gravidade do prolapso e a capacidade de reter o mesmo na vagina. Além destes parâmetros de escolha é importante que a paciente ou sua cuidadora tenham facilidade em manipula-los para a sua higiene.

Quando o procedimento cirúrgico for contraindicado, o pessário tem a vantagem de poder ser colocado e retirado pela própria paciente, quando devidamente orientada, não influenciando negativamente em seu convívio social, trabalho e atividade sexual.
Porém, em alguns casos podem ocorrer desconfortos vaginais, erosões, sangramentos e corrimentos ${ }^{4}$, sendo por isso fundamentais os cuidados com higienização adequada, assim como o treinamento supervisionado por fisioterapeuta, enfermeira ou assistente social, com apoio de um familiar se possível para a sua retirada, tempo de descanso e reintrodução.

Outro fator que deve ser levado em consideração na descontinuidade do uso do pessário, é a incontinência urinária, que pode ocorrer após a correção do prolapso. Muitas mulheres, retornam com o sintoma que precisa ser avaliado adequadamente para a indicação de tratamento adequado (fisioterapia, medicamentoso ou cirúrgico) ${ }^{2}$. Para isto, é utilizado o Estudo Urodinâmico, que tem por finalidade classificar o tipo de incontinência urinária (urgência, esforço ou mista), que em conjunto com o exame clínico torna o diagnóstico mais fidedigno para iniciar o tratamento.

Existem trabalhos que discorrem sobre o uso do pessário como tratamento conservador para POP, e foram publicadas evidências de que este método terapêutico é eficaz na melhora da QV e função sexual das pacientes, bem como na diminuição dos sintomas vaginais ${ }^{5}$. Entretanto a literatura carece de estudos com a utilização de questionários validados e específicos para o prolapso, para avaliar a QV, antes, durante e depois do uso do pessário. Assim, o objetivo dessa revisão foi analisar os trabalhos existentes, com uso de pessário em mulheres com prolapso de órgãos pélvicos relacionado à melhora da $\mathrm{QV}$.

\section{MÉTODO}

Para uma pesquisa completa foram utilizadas as etapas PRISMA. Os artigos foram pesquisados no mês de janeiro de 2017 a julho de 2017, utilizando as Bases de Dados Scientific Electronic Library On-line (SciELO) e PubMed", com termos "POP and pessary, POP and Quality of life". Como critérios de inclusão utilizamos estudos publicados entre janeiro de 2011 e dezembro de 2016 em inglês e português que abordassem POP, uso de pessário e QV. Como critério de exclusão os estudos que não utilizaram questionários de QV ou que não estivessem validados, pacientes com sintomas de incontinência urinária, sintomas intestinais e publicações anteriores a janeiro de 2011.

\section{RESULTADOS}

Foram encontrados 25 artigos com os termos $\mathrm{MeSH}$, pessary and POP, POP and QV, na base de dados da PubMed e nenhum artigo foi encontrado com estes termos no SciELO. O fluxo de informações da pesquisa realizada nas bases de dados, estão apresentados no fluxograma (Figura 1).

Após aplicação dos critérios de exclusão, restaram 10 artigos que foram utilizados nesta revisão (Tabela 1). 
Bases de Dados PubMed ( $\mathrm{n}=25)$

\section{Bases de Dados SciELO $(\mathrm{n}=0)$}

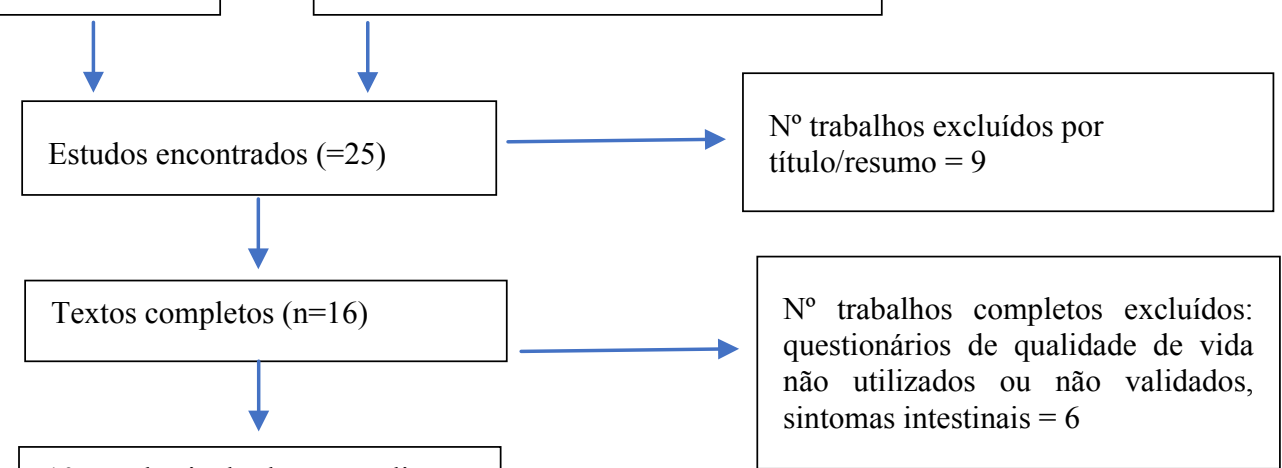

10 estudos incluídos na análise qualitativa

Figura 1. Fluxograma com estudos encontrados nas bases de dados

Tabela 1 - Resumo dos 10 artigos publicados sobre uso de pessários e QV

\begin{tabular}{|c|c|c|c|c|c|c|c|}
\hline Referência & $\begin{array}{l}\text { Desenho } \\
\text { estudo }\end{array}$ & Idade & Paridade & $\begin{array}{l}\text { Questionário } \\
\text { utilizado }\end{array}$ & $\begin{array}{l}\text { Tempo de } \\
\text { seguimento }\end{array}$ & $\begin{array}{l}\text { Descontinuidade } \\
\text { pessário }\end{array}$ & Resultados \\
\hline $\begin{array}{l}\text { de Albuquerque } \\
\text { Coelho et al. }{ }^{4}\end{array}$ & $\begin{array}{l}\text { Revisão } \\
\text { sistemática }\end{array}$ & \pm 75 & NR & $\begin{array}{l}\text { POPIQ, } \\
\text { CRAIQ, UIQ }\end{array}$ & 6 meses & $\begin{array}{l}\text { Desconforto, } \\
\text { expulsão do } \\
\text { dispositivo }\end{array}$ & $\begin{array}{l}\text { O uso de pessário trouxe } \\
\text { benefícios na qualidade de } \\
\text { vida e mulheres relataram } \\
\text { níveis bons de satisfação. }\end{array}$ \\
\hline $\begin{array}{l}\text { Manchana; } \\
\text { Bunyavejchevin }{ }^{5}\end{array}$ & $\begin{array}{l}\text { Estudo } \\
\text { prospectivo }\end{array}$ & \pm 67 & NR & P-QOL & 1 ano & Não apresentado & $\begin{array}{l}\text { Melhora dos sintomas } \\
\text { e qualidade de vida foi } \\
\text { relatada pelas pacientes }\end{array}$ \\
\hline Lone et al. ${ }^{6}$ & Prospectivo & $\begin{array}{l} \pm 67 \\
\text { pessário } \\
\pm 59 \\
\text { cirurgias }\end{array}$ & \pm 5 & $\begin{array}{l}\text { ICIQ-VS e } \\
\text { ICIQ-UI (SF) }\end{array}$ & 1 ano & $\begin{array}{l}\text { Desconforto, } \\
\text { dificuldade na } \\
\text { colocação }\end{array}$ & $\begin{array}{l}\text { Não houve diferença } \\
\text { significativa entre os } \\
\text { dois tipos de tratamento } \\
\text { relacionado a Qualidade } \\
\text { de Vida }\end{array}$ \\
\hline Lamers et al. ${ }^{7}$ & $\begin{array}{l}\text { Revisão } \\
\text { sistemática }\end{array}$ & NR & \pm 4 & $\begin{array}{l}\text { KHQ, FSFI, } \\
\text { PFIQ }\end{array}$ & $\begin{array}{l}\text { Média de } 1 \\
\text { ano }\end{array}$ & $\begin{array}{l}\text { Constipação, } \\
\text { desconforto } \\
\text { vaginal, erosões, } \\
\text { sangramento } \\
\end{array}$ & $\begin{array}{l}\text { Houve eficácia no uso do } \\
\text { pessário relacionado com } \\
\text { a qualidade de vida }\end{array}$ \\
\hline Ko et al. ${ }^{8}$ & $\begin{array}{l}\text { Estudo } \\
\text { retrospectivo }\end{array}$ & NR & NR & UDI-6, IIQ-7 & 1 ano & $\begin{array}{l}\text { Incontinência } \\
\text { urinária stress, } \\
\text { sem apoio } \\
\text { familiar }\end{array}$ & $\begin{array}{l}\text { Achados de melhora nos } \\
\text { sintomas de prolapso } \\
\text { e consequentemente } \\
\text { aumento a qualidade de } \\
\text { vida }\end{array}$ \\
\hline Tenfelde et al. ${ }^{9}$ & $\begin{array}{l}\text { Estudo } \\
\text { retrospectivo }\end{array}$ & \pm 66 & NR & $\begin{array}{l}\text { PFIQ-7, } \\
\text { PFDI-20 }\end{array}$ & $\begin{array}{l}6 \text { a } 12 \\
\text { meses }\end{array}$ & $\begin{array}{l}\text { Dificuldade na } \\
\text { colocação }\end{array}$ & $\begin{array}{l}\text { Os três grupos (bem } \\
\text { sucedido, mal sucedido } \\
\text { e dificuldade no encaixe } \\
\text { do pessário) não } \\
\text { apresentaram diferenças } \\
\text { clínicas tais como idade, } \\
\text { cirurgia prévia, sendo o } \\
\text { motivo de descontinuidade } \\
\text { a dificuldade na colocação } \\
\text { do pessário, O grupo } \\
\text { bem sucedido apresentou } \\
\text { melhora nos resultados } \\
\text { dos questionários. }\end{array}$ \\
\hline Chan et al. ${ }^{10}$ & $\begin{array}{l}\text { Estudo } \\
\text { transversal } \\
\text { observacional }\end{array}$ & $\pm 74,4$ & NR & $\begin{array}{l}\text { PFDI, PFIQ, } \\
\text { Short Form- } \\
36 \text { (SF-36) }\end{array}$ & 4 a 6 meses & $\begin{array}{l}\text { Sangramento } \\
\text { e desconforte } \\
\text { vaginal }\end{array}$ & $\begin{array}{l}\text { As complicações com o } \\
\text { pessário (desconforto, } \\
\text { erosão) foram fatores para } \\
\text { a escolha cirúrgica nos } \\
\text { questionários de qualidade } \\
\text { de vida. }\end{array}$ \\
\hline
\end{tabular}


Barros CR, et al. Tratamento conservador de prolapso de órgão pélvico com pessário.

Tabela 1 - Resumo dos 10 artigos publicados sobre uso de pessários e QV

Continuação

\begin{tabular}{|c|c|c|c|c|c|c|c|}
\hline Referência & $\begin{array}{l}\text { Desenho } \\
\text { estudo }\end{array}$ & Idade & Paridade & $\begin{array}{l}\text { Questionário } \\
\text { utilizado }\end{array}$ & $\begin{array}{l}\text { Tempo de } \\
\text { seguimento }\end{array}$ & $\begin{array}{l}\text { Descontinuidade } \\
\text { pessário }\end{array}$ & Resultados \\
\hline Wang et al. ${ }^{11}$ & $\begin{array}{l}\text { Estudo } \\
\text { Prospectivo } \\
\text { Coorte }\end{array}$ & \pm 66 & NR & $\begin{array}{l}\text { SF-12 e } \\
\text { PFIQ-7 }\end{array}$ & 3 e 6 meses & $\begin{array}{l}\text { Desconforto, } \\
\text { retenção urinária, } \\
\text { tamanho } \\
\text { inadequado }\end{array}$ & $\begin{array}{l}\text { Em comparação com os } \\
\text { achados iniciais, o SF-12 } \\
\text { e o PFIQ-7 demonstraram } \\
\text { melhora significativa na } \\
\text { QV. }\end{array}$ \\
\hline Wang et al. ${ }^{12}$ & $\begin{array}{l}\text { Estudo } \\
\text { comparativo }\end{array}$ & NR & NR & PGI-I & 4 a 5 meses & $\begin{array}{l}\text { Secreção, disúria, } \\
\text { incontinência }\end{array}$ & $\begin{array}{l}\text { O grupo cirúrgico } \\
\text { apresentou mais } \\
\text { resultado, porém o } \\
\text { PGI-I não mostrou } \\
\text { diferença estatisticamente } \\
\text { significante, sendo que } \\
\text { todas apresentaram } \\
\text { melhora do score de QV. }\end{array}$ \\
\hline Yang et al. ${ }^{13}$ & $\begin{array}{l}\text { Observacional } \\
\text { Transversal }\end{array}$ & NR & NR & $\begin{array}{l}\text { PFDI-20-SF) } \\
\text { e o Pelvic } \\
\text { Floor Impact } \\
\text { Questionnaire. }\end{array}$ & 4,5 anos & Erosão vaginal & $\begin{array}{l}\text { A maioria das mulheres } \\
\text { retornaram a cada } 3 \\
\text { meses para verificação } \\
\text { do pessário e não houve } \\
\text { diferença significativa } \\
\text { entre os grupos. }\end{array}$ \\
\hline
\end{tabular}

POPIQ Pelvic Organ Prolapse Impact Questionnaire, CRAIQ Colorectal-Anal Impact Questionnaire, UIQ Urinary Impact Questionnaire, KHQ King's Health Questionnaire, FSFI Female Sexual Function Index, PFIQ Pelvic Floor Impact Questionnaire, ICIQ-VS International Consultation on Incontinence Questionnaire Vaginal Symptoms, ICIQ-UI(SF), Urinary Incontinence Short Form, PFDI-20-SF Pelvic Floor Distress Inventory Short Form, SF-12 Short Form Health Survey, PFIQ-7 Pelvic Floor Impact Questionnaire Short Form, Short Form-36 Questionário de Qualidade de Vida, P-QOL Prolapse Quality of Life, PGI-I Patient Global Impression of Improvement, NR Não Relatado.

Nos artigos selecionados foi possível verificar que a faixa etária, tempo de seguimento foram semelhantes com relação aos tratamentos cirúrgicos, porém o uso de pessário pode ter descontinuidade. Os resultados mostram que em todos os estudos o pessário obteve boa evolução, melhorando sintomas vaginais e de prolapso, assim como nos pacientes que realizaram a cirurgia. Os questionários utilizados foram diversificados, mas todos englobavam uroginecologia e o impacto da QV. Apenas um estudo utilizou o SF-36 que é um questionário geral de QV e apenas um estudo utilizou um questionário específico para prolapso - PQOL.

As intervenções com uso de pessário tiveram a escolha do modelo em anel em 11 estudos, donut e anel em 2 estudos e em apenas um estudo foi utilizado o modelo Gellhorn. O tempo médio de seguimento das pesquisas foi de 6 meses em $50 \%$ dos estudos, 1 ano em $28,57 \%$ dos estudos e apenas 3 meses em 21,42\% deles.

Importante ressaltar que nos estudos com mais de 6 meses de seguimento ocorreram os melhores resultados, pois neste período ocorreu boa adaptação do pessário, sendo possível quantificar de maneira mais fidedigna a melhora dos sintomas vaginais e de QV, devido ao acompanhamento durante todo o tratamento com o dispositivo, sendo possível tirar dúvidas e solucionar possíveis desconfortos e complicações o que gera maior segurança e adaptação ao dispositivo.

O uso do estrogênio na forma de creme vaginal foi utilizado apenas quando havia atrofia vaginal sendo ele importante para minimizar o risco de erosões vaginais. Com relação a descontinuidade com o tratamento conservador, os fatores mais apontados em $12 \%$ dos casos foram a dificuldade em colocação (devido ao não conhecimento da anatomia ou falta de apoio familiar) e desconforto vaginal, sendo a incontinência urinária observada em apenas em $10 \%$ dos casos. Pacientes que não tem suporte familiar são mais propensas a desistência e já o fator incontinência urinária, se deve a correção do prolapso, necessitando de acompanhamento fisioterapêutico ou cirúrgico para avaliar e tratar o sintoma.

\section{DISCUSSÃO}

Segundo Lone et al. ${ }^{6}$ o uso do pessário mostrou-se eficaz na resolução dos sintomas de POP, não apresentando desempenho inferior ao método cirúrgico de correção. As complicações mais frequentes e que em alguns casos foi o motivo para a descontinuidade do tratamento, foram desconforto vaginal, erosões, dificuldade na colocação, sangramentos e em alguns casos incontinência urinária após redução do prolapso ${ }^{7}$.

Mesmo em estágios mais avançados de prolapso (Grau 3 ou 4), o pessário mostrou-se eficaz reduzindo os sintomas e consequentemente melhorando a qualidade de vida em $28 \%$ dos casos no decorrer de um ano de uso do dispositivo ${ }^{5}$.

Estudos com seguimento de um ano relataram que as mulheres idosas tiveram maior aceitação no uso de pessário do que mulheres mais jovens, principalmente as 
sexualmente ativas e foi possível verificar que em alguns casos a descontinuidade do uso do dispositivo se deu pela falta de suporte familiar, fisioterapêutico, enfermagem ou de assistente social ou ainda de incontinência urinária ${ }^{8,13}$.

A equipe multiprofissional é importante para que as pacientes tenham suporte e treinamento adequado para a colocação e recolocação do pessário, necessitando do apoio familiar para o sucesso da terapia.

Importante ressaltar que as pacientes dos estudos analisados tiveram uma média de 50-67 anos de idade, o que difere da nossa população atendida nos postos de saúde e ambulatórios de assoalho pélvico SUS no Brasil na qual a idade inicial média é de 70-90 anos ${ }^{4}$. Isto nos mostra a necessidade de mais estudos com essa população, afim de verificar os resultados com o uso do pessário visto que os prolapsos tendem a ser maiores (Grau 3 e 4), acompanhados de atrofia vaginal e necessidade de um familiar ou cuidador para auxiliar na colocação e retirada do dispositivo.

A multiparidade também é um fator determinante para a ocorrência de prolapsos nas mulheres, sendo que, os estudos realizados em outros países que compõe a amostra analisada, referem naquelas uma média de 3-5 partos enquanto que as mulheres com estes sintomas no Brasil referem uma média de 5-8 partos alguns dos quais com utilização de fórcipe ou até de partos realizados em ambiente domiciliar'.

Vale ressaltar também que mesmo nos estudos

\section{REFERÊNCIAS}

1. Haylen BT, de Ridder D, Freeman RM, Swift SE, Berghmans B, Lee J, Monga A, Petri E, Rizk DE, Sand PK, Schaer GN. An International Urogynecological Association (IUGA)/ International Continence Society (ICS) joint report on the terminology for female pelvic floor dysfunction. Int Urogynecol J. 2010;21(1):5-26. doi: 10.1007/s00192-0090976-9.

2. Castello Girão MJB, et al. Tratado de uroginecologia e disfunções do assoalho pélvico. Barueri, SP: Manole; 2015.

3. Oliveira JC, Albuquerque FRPC, Lins IB. Projeção da população do Brasil por sexo e idade para o período de 1980-2050: revisão de 2004: Metodologia e resultados: Estimativas anuais e mensais da população do Brasil e das unidades da federação: 1980-2020: metodologia; estimativas das populações municipais: Metodologia. Rio de Janeiro: IBGE; 2004. Disponível em: http //www.ibge.gov.br/ home/ estatística/ população/estimativa2004/metodologia.pdf.

4. de Albuquerque Coelho SC, de Castro EB, Juliato CR. Female pelvic organ prolapse using pessaries: systematic review. Int Urogynecol J. 2016;27(12):1797-803. doi: 10.1007/s00192016-2991-y.

5. Manchana T, Bunyavejchevin S. Impact on quality of life after ring pessary use for pelvic organ prolapse. Int Urogynecol J. 2012;23(7):873-7. doi: 10.1007/s00192-011-1634-6.

6. Lone F, Thakar R, Sultan AH. One-year prospective comparison of vaginal pessaries and surgery for pelvic organ utilizando questionários diversificados e muitos não exclusivos de prolapso, foi possível observar resultados positivos. A maioria das pacientes com descontinuidade no uso, foi relacionada com a dificuldade na colocação do pessário ou em alguns casos na ocorrência de incontinência urinária $^{11,12}$.

O questionário mais utilizado foi o Pelvic Floor Impact Questionnaire (PFIQ), que compreende três escalas: Urinary Distress Inventory, Colorectal Distress Inventory e Pelvic Organ Prolapse Distress Inventory ${ }^{7,9,10,11}$. $\mathrm{O}$ questionário $\mathrm{P}-\mathrm{QOL}$ que estuda especificamente a qualidade de vida pós tratamentos em portadoras de prolapsos de órgãos pélvicos infelizmente foi aplicado em apenas um estudo de nossa amostra ${ }^{5}$.

Desta maneira fazem-se necessários estudos utilizando o questionário P-QOL, para verificar o impacto da QV estritamente relacionado ao POP, possibilitando obter-se scores específicos do tratamento conservador com pessário, por ser este dispositivo de baixo custo, sendo extremamente importante no Sistema Único de Saúde.

\section{CONCLUSÃO}

Podemos concluir que o pessário é uma opção viável de tratamento para mulheres com POP, mesmo em estadios avançados (Grau 3 e 4), que impactam positivamente na $\mathrm{QV}$, sendo eles especialmente indicados em pacientes que não desejem ou não possam ser cirurgicamente tratadas.

prolapse using the validated ICIQ-VS and ICIQ-UI (SF) questionnaires. Int Urogynecol J. 2015;26(9):1305-12. doi: 10.1007/s00192-015-2686-9.

7. Lamers BH, Broekman BM, Milani AL. Pessary treatment for pelvic organ prolapse and health-related quality of life: a review. Int Urogynecol J. 2011;22(6):637-44. doi: 10.1007/ s00192-011-1390-7.

8. Ko PC, Lo TS, Tseng LH, Lin YH, Liang CC, Lee SJ. Use of a pessary in treatment of pelvic organ prolapse: quality of life, compliance, and failure at 1-year follow-up. J Minim Invasive Gynecol. 2011;18(1):68-74. doi: 10.1016/j.jmig.2010.09.006.

9. Tenfelde S, Tell D, Thomas TN, Kenton K. Quality of life in women who use pessaries for longer than 12 months. Female Pelvic Med Reconstr Surg. 2015;21(3):146-9. doi: 10.1097/ SPV.0000000000000154.

10. Chan SS, Cheung RY, Yiu KW, Lee LL, Pang AW, Chung TK. Symptoms, quality of life, and factors affecting women's treatment decisions regarding pelvic organ prolapse. Int Urogynecol J. 2012;23(8):1027-33. doi:10.1007/s00192-012$1698-y$.

11. Wang XQ, Zhu L, Li B, Tang X, Xu T. A prospective study of pessary treatment for pelvic organ prolapse and health-related quality-of-life. Zhonghua Yi Xue ZaZhi. 2013;93(37):2982-5.

12. Wang Y, Han J, Zhang K, Zhu F, Yang J, Wang Y. Relevance between expectations before treatment, new symptoms and satisfaction after treatment in patients with pelvic organ prolapse. Zhonghua Fu Chan Ke Za Zhi. 2015;50(9):664-7. 
Barros CR, et al. Tratamento conservador de prolapso de órgão pélvico com pessário.

13. Yang JF, Han JS, Zhu FL, Wang YT, Yao Y, Qiao J. Clinical study on silicone pessary in the treatment of pelvic organ prolapse. Zhonghua Fu Chan Ke Za Zhi. 2012;47(7):487-91.

14. Hutton B, Salanti G, Caldwell DM, Chaimani A, Schmid $\mathrm{CH}$, Cameron C, Ioannidis JP, Straus S, Thorlund K, Jansen

Recebido em: 08.01.18

Aceito em: 01.03 .18
JP, Mulrow C, Catalá-López F, Gøtzsche PC, Dickersin K, Boutron I, Altman DG, Moher D. The PRISMA extension statement for reporting of systematic reviews incorporating network meta-analyses of health care interventions: checklist and explanations. Ann Intern Med. 2015;162(11):777-84. doi: 10.7326/M14-2385. 